\title{
NOTE ON THE APPLICATION OF THE METHOD OF HARMONIC ANALYSIS.
}

BY

\section{Y. Tsuiji.}

5. Determination of Constants:--Let us consider the determination of the amplitudes and phase angles of the periodic function by means of the Method of the Least Squares

Substitute $\overline{\bar{y}}$ for $\frac{1}{m} \sum_{\mu=0}^{m-1} f\left(t_{1}+\mu \frac{\tau}{m}\right)$, then the formula (A) in $\$ 4$ becomes

$$
\overline{\bar{y}}=a_{0}+\sum_{n=1} \bar{a}_{n} \sin \left\{\frac{\grave{2} n \pi}{T}\left(t_{1}+\frac{m-1}{2 m} \tau\right)+\varphi_{n}\right\}
$$

By expansion we obtain

$$
\overline{\bar{y}}=a_{0}+\sum_{n \rightarrow 1} p_{n} \cos \frac{2 n \pi}{T}\left(t_{1}+\frac{m-1}{2 m} \tau\right)+\sum_{n=1} q_{n} \sin \frac{2 \mathrm{n} \pi}{\mathrm{T}}\left(t_{1}+\frac{m-1}{2 m} \tau\right),
$$

in which $p_{n}=\overline{\bar{a}}_{n} \sin \varphi_{n}$,

and $\quad q_{n}=\overline{\bar{a}}_{n} \cos \varphi_{n}$.

Now there are $\frac{T}{\tau}$ groups in the one complete cycle of the phenomenon. Hence we have the following $\frac{T}{\tau}$ equations :

$$
\begin{gathered}
a_{0}+\sum_{n=1} p_{n} \cos \frac{2 n \pi}{\mathrm{T}} \frac{m-1}{2 m} \tau+\sum_{n=1} q_{n} \sin \frac{2 n \pi}{\mathrm{T}}-\frac{m-1}{2 m} \tau-\overline{\bar{y}}_{1}=0 \\
a_{0}+\sum_{n=1} p_{n} \cos \frac{2 n \pi}{\mathrm{T}}-\frac{3 m-1}{2 m} \tau+\sum_{n=1} q_{n} \sin \frac{2 n \pi}{\mathrm{T}} \frac{3 m-1}{2 m} \tau-\bar{y}_{2}=0 \\
\text { etc. etc. }
\end{gathered}
$$


$a_{0}+\sum_{n=1} p_{n} \cos \frac{2 n \pi}{\mathrm{T}} \frac{2-\frac{-1}{2} m-1}{2 m}++\sum_{n_{i}=1} q_{r_{b}} \sin \frac{2 n \pi}{\mathrm{T}} \cdot \frac{(2 \nu-1) m-1}{2 m}-\overline{\bar{y}}_{\nu}=0$ etc. etc.

$a_{0}+\sum_{n=1} p_{n} \cos \frac{2 n \pi}{\mathrm{T}}\left(\frac{\mathrm{T}}{\tau}-\frac{m+1}{2 m}\right)=+\sum_{n=1} q_{n} \sin \frac{2 n \pi}{\mathrm{T}}\left(\frac{\mathrm{T}}{\tau}-\frac{m+1}{2 m}\right) \tau-\overline{\bar{y}}_{\frac{\mathrm{T}}{\tau}}=0$.

in which $\bar{y}_{1}, \bar{y}_{2}, \bar{y}_{3}, \ldots . \bar{y}_{\frac{x}{\tau}}$ are respectively the mean values of the successive groups. From these equations of condition we obtain the normal equations as follows :-

$$
\begin{aligned}
& \frac{\mathrm{T}}{\tau} a_{o}+\sum_{h=1}\left[\mathrm{C}_{h v}\right] p_{h}+\sum_{h=1}\left[\mathrm{~S}_{h t}\right] q_{h}-[\vec{y}]=0, \\
& \text { ctc. etc. } \\
& {\left[\mathrm{C}_{n v}\right] a_{0}+\sum_{n=1}\left[\mathrm{C}_{h \nu} \mathrm{C}_{n k}\right] p_{k}+\sum_{n=1}\left[\mathrm{~S}_{h v} \mathrm{C}_{n \nu}\right] q_{n}-\left[\overline{\bar{y}}_{\nu} \mathrm{C}_{n \nu}\right]=0} \\
& \text { etc. etc. } \\
& \begin{array}{c}
{\left[\mathrm{S}_{n, s}\right] a_{s}+\sum_{n=1}\left[\mathrm{~S}_{n \nu} \mathrm{C}_{i \nu}\right] p_{n}+\sum_{n=1}\left[\mathrm{~S}_{i \nu} \mathrm{C}_{n \nu}\right] q_{n}-\left[\overline{\bar{y}}_{\nu} \mathrm{S}_{n \nu}\right]=0,} \\
\text { etc, etc. }
\end{array}
\end{aligned}
$$

where $\quad S_{h \nu}=\sin \frac{2 \mathrm{~h} \pi}{\mathrm{T}} \frac{(2 \nu-1) m-1}{2 m}-$

$$
\mathrm{C}_{h \nu}=\cos \frac{2 h \pi}{\mathrm{T}} \frac{(2 \nu-1) m-1}{2 m}-
$$

and

$$
\left[\mathrm{S}_{h \nu}\right]=\sum_{\nu=1}^{\frac{T}{T}} S_{H_{\nu}},\left[\mathrm{S}_{h_{\nu}} \mathrm{C}_{n_{\nu}}\right]=\sum_{\nu=1}^{\frac{T}{\tau}} \mathrm{S}_{h \nu} \mathrm{C}_{n \nu} .
$$

$$
\text { etc., etc. }
$$

It is easy to show that all of the summations of the series of sines, of cosines and of thier products in the last equations become null, except

$$
\left[\mathrm{S}_{n_{\nu}} \mathrm{S}_{n_{\nu}}\right] \text { and }\left[\mathrm{C}_{n_{\nu}} \mathrm{C}_{n_{\nu}}\right. \text { l. }
$$

both of which have the same value

$$
\frac{1}{2} \frac{T}{\tau}
$$


Hence we get. for the normal equations:

$$
\begin{aligned}
& \frac{T}{\tau} a_{\circ}-[\bar{y}]=0, \\
& \frac{1}{2} \frac{T}{\tau} p_{n}-\left[\overline{\overline{y_{\nu}}} \mathrm{C}_{n_{\nu}}\right]=0, \\
& \frac{1}{2} \frac{\mathrm{T}}{\tau} q_{n}-\left[\overline{\bar{y}_{\nu}} \mathrm{S}_{n_{\nu}}\right]=0 .
\end{aligned}
$$$$
\}
$$

Therefore we obtain

$$
\begin{aligned}
& a_{0}=\frac{\tau}{T}\left(\overline{\overline{y_{1}}}+\overline{\bar{y}}_{2}+\cdots \cdots+\overline{\overline{y_{z}}}\right) \text {, }
\end{aligned}
$$

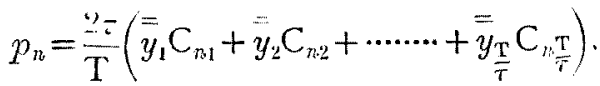

$$
\begin{aligned}
& q_{n}=\frac{2 \tau}{T}\left(\overline{\bar{y}}_{1} S_{n 1}+\overline{\bar{y}}_{2} \mathrm{~S}_{n^{2}}+\cdots \cdots \cdots+\overline{\bar{y}}_{\frac{\mathrm{T}}{\tau}} \mathrm{S}_{n \frac{\mathrm{T}}{\tau}}\right) \text {. }
\end{aligned}
$$

and hence

$$
\overline{\bar{a}}_{n}=\sqrt{p_{n b}^{2}+q_{n b}^{2}} \text { and } \tan \varphi_{n}=\frac{p_{n}}{q_{n}},
$$

which are required.

Remark :- The functional expression of $\bar{y}$ may be expanded in another way, for example:

$$
\bar{y}=a_{0}+\sum_{n=1} p_{n} \cos \frac{2 n \pi}{\mathrm{T}}\left(t_{1}+\frac{\tau}{2}\right)+\sum_{n=1} q_{n} \sin \frac{2 n \pi}{\mathrm{T}}\left(t_{1}+\frac{\bar{\tau}}{2}\right)
$$

in which $\quad p_{n}^{\prime}=\vec{a}_{n} \sin \left(\varphi_{n}-\frac{n \pi \tau}{\mathrm{T} m}\right)$,

and

$$
q_{n}=\bar{a}_{n} \cos \left(\varphi_{n}-\frac{n \pi \tau}{\mathrm{T} m}\right) \text {. }
$$

Let us put $S_{{ }^{\prime}}=\sin \frac{2 n \pi}{T} \frac{2 \nu-1}{2} \tau$,

and

$$
\mathrm{C}^{\prime}{ }^{\prime}=\cos \frac{2 n \pi}{\mathrm{T}} \frac{2 \nu-1}{2} \tau \text {. }
$$


Then, by the similar processes as above we obtain

$$
\begin{aligned}
& a_{0}=\frac{\tau}{\mathrm{T}}\left(\overline{\bar{y}}_{1}+\overline{\bar{y}}_{2}+\cdots \cdots+\overline{\bar{y}}_{\frac{\mathrm{T}}{\tau}}\right), \\
& p_{n}^{\prime}=\frac{2 \tau}{\mathrm{T}}\left(\overline{\bar{y}}_{1} \mathrm{C}_{n 1}^{\prime}+\overline{\bar{y}}_{2} \mathrm{C}_{n 2}^{\prime}+\cdots \cdots+\overline{\bar{y}}_{\frac{\mathrm{T}}{\tau}} \mathrm{C}_{n}^{\prime} \frac{\mathrm{T}}{\bar{\tau}}\right), \\
& q_{n}^{\prime}=\frac{2 \tau}{\mathrm{T}}\left(\overline{\bar{y}}_{1} \mathrm{~S}_{n 1}^{\prime}+\overline{\bar{y}}_{2} \mathrm{~S}_{n 2}^{\prime}+\cdots \cdots+\overline{\bar{y}}_{\frac{\mathrm{T}}{\tau}} \mathrm{S}_{n}^{\prime} \frac{\mathrm{T}}{\tau}\right), \\
& \overline{\bar{a}}_{n}=\sqrt{p_{n}^{\prime 2}+\bar{q}_{n}^{\prime \prime}}, \tan \left(\varphi_{n}-\frac{n \pi}{\mathrm{T}} \frac{\tau}{m}\right)=\frac{p_{n}^{\prime}}{q_{n}^{\prime}} .
\end{aligned}
$$

and

6. Literature:-When the first part of the present memoir was passing through the press and was ready to appear on last subscription of this Journal* I was kindly called attension by Dr. $T$. Okada to what the idea expressed in the introductory paragraph is old one and to what Prof. C. G. Knot had already treated on this problem mathematically in his excellent treatise on "Physics of the Earthquake Phenomena."** His discussion is concluded with reference to the correction of the amplitude of the earthquake frequency determined by means of the overlapping summation.

Thus the latter part (paragraph4) and a little of the other of my note are found free from overlapping on Prof. Knot's work.

In conclusion I wish to express the heartiest thanks to Dr. T. Okada for his kind guidance.

$$
\text { May 28, } 1912 .
$$

Sericultural College, Uyeda, Japan.

* Y. Tsuiji :--Journal of the Met. Soc. of Japan, 3ryear, No. 5, May rgra.

** C. G. Knot, :-Physics of the Earthqualse / henomena, pp. IrI-ri2. The original of which is: C. G. Knot:-Earthquake Frequency. Transactions of the Seismological Society of Japan. Vol. IX, Part I, ISS6, p. 20. 